Alexis Joachimides, Stephanie Milling,

Ilse Müllner, Yvonne Sophie Thöne (Hg.)

Opfer - Beute - Hauptgericht 

Alexis Joachimides, Stephanie Milling,

Ilse Müllner, Yvonne Sophie Thöne (Hg.)

Opfer - Beute - Hauptgericht

Tiertötungen im interdisziplinären Diskurs

[transcript $]$ 
Dieser Band ist im Zusammenhang mit den Forschungen des LOEWE-Schwerpunkts »Tier - Mensch - Gesellschaft: Ansätze einer interdisziplinären Tierforschung « an der Universität Kassel entstanden. Die Drucklegung wurde durch Mittel der Landes-Offensive zur Entwicklung Wissenschaftlich-ökonomischer Exzellenz (LOEWE) des Hessischen Ministeriums für Wissenschaft und Kunst ermöglicht.

\section{SLEWE}

Exzellente Forschung für Hessens Zukunft

\section{Bibliografische Information der Deutschen Nationalbibliothek}

Die Deutsche Nationalbibliothek verzeichnet diese Publikation in der Deutschen Nationalbibliografie; detaillierte bibliografische Daten sind im Internet über http://dnb.d-nb.de abrufbar.

\section{두 2016 transcript Verlag, Bielefeld}

Die Verwertung der Texte und Bilder ist ohne Zustimmung des Verlages urheberrechtswidrig und strafbar. Das gilt auch für Vervielfältigungen, Übersetzungen, Mikroverfilmungen und für die Verarbeitung mit elektronischen Systemen.

Umschlaggestaltung: Kordula Röckenhaus, Bielefeld Umschlagabbildung: Assyrisches Palastrelief, 7. Jh. v. Chr.;

Relief aus: Hieke, Thomas: Levitikus I-I5

Korrektorat: Dr. Frank Hermenau

Satz: Peter Oberhuber | Visuelle Kommunikation, www.peteroberhuber.de

Printed in Germany

Print-ISBN 978-3-8376-3507-2

PDF-ISBN978-3-8394-3507-6

Gedruckt auf alterungsbeständigem Papier mit chlorfrei gebleichtem Zellstoff. Besuchen Sie uns im Internet: $h t t p: / / w w w . t r a n s c r i p t-v e r l a g . d e$

Bitte fordern Sie unser Gesamtverzeichnis und andere Broschüren an unter: info@transcript-verlag.de 


\title{
Das Geschlecht der Opfertiere
}

\author{
Überlegungen anhand von Opferbestimmungen der Tora
}

Ilse Müllner

\section{Tora: ERzählung Und Weisung}

Im biblischen Israel gehört das Opfern von Tieren zu den basalen rituellen Ausdrucksformen, mit denen Menschen in eine Beziehung zum Göttlichen treten. Auch die Erinnerung an das Grunddatum der Geschichte Israels - den Exodus - in der Pessachfeier ist mit der ritualisierten Tötung von Tieren verbunden. Ob das Schlachten des Pessach-Lamms wirklich als Opfer zu qualifizieren ist, bleibt strittig. ${ }^{I}$ Unstrittig aber ist, dass die Schlachtung eines »fehlerfreien, männlichen, einjährigen Lamms, des Jungen eines Schafes oder einer Ziege« (Ex I2,5) zum erzählten Auszug aus Ägypten ebenso gehört wie zu dessen ritualisiertem Gedenken. Pessach ist für die jüdische Identität nicht irgendein, sondern das konstitutive Grunddatum. Die Beziehung zwischen dem jüdischen Volk und seinem Gott $\mathrm{JHWH}^{2}$ wird in diesem Ereignis des Auszugs aus der ägyptischen Sklaverei in das verheißene Land grundgelegt. Das ritualisierte Gedenken dieser Befreiungstat bestimmt bis in die Gegenwart über den Festkalender auch den Jahresrhythmus und konkretisiert sich in der jährlichen Feier des Pessach. Bereits in der ersten Erzäh-

1 | Vgl. C. Dohmen: Exodus, S. 291-294.

2 Der Gottesname, der mit dem hebräischen Tetragramm יהוה (JHWH) bezeichnet wird, wurde(und wird) im Judentum schon relativ früh nicht ausgesprochen, sondern mit Ersatzbegriffen bezeichnet, sodass der Name bereits in der griechischen Bibelübersetzung (ab dem 3. Jh. v.u.Z.) durch kyrios (Herr) wiedergegeben wurde. Viele Bibelübersetzungen geben das Tetragramm in dieser Tradition stehend mit "Herr" oder auch "HERR" wieder. Die ursprüngliche Aussprache ist aufgrund der ursprünglich vokallosen hebräischen Schrift unklar, kann aber anhand griechischer Umschriften als "Jahwe " rekonstruiert werden. In der hebräischen Bibel wird der Namen allerdings so vokalisiert, dass damit auf den Ersatzbegriff 'adonāj verwiesen wird. Deshalb wird in der exegetischen Literatur häufig nur das vokallose Tetragramm JHWH zur Wiedergabe des Gottesnamens verwendet. S. Becking, Jahwe. 
lung des Auszugsereignisses im Buch Exodus, in den Kapiteln I2-I5, wird das Narrativ des Ereignisses mit Anweisungen zur Durchführung des Ritus verschränkt. ${ }^{3}$

"Der Gesamtzusammenhang dieser vier Kapitel ist als eine fortlaufende Erzählung gestaltet, aber unterhalb dieser Ebene ändern sich die literarischen Formen mehrfach, anders formuliert: In einen narrativen Rahmen werden unterschiedliche Gattungen integriert, so dass ein komplexes literarisches Gebilde entsteht. " ${ }^{4}$

Sowohl durch Hymnen als auch - und darum geht es an dieser Stelle - durch Ritualanweisungen wird die Sequentialität des Erzählens durchbrochen. Man könnte sich einen Text vorstellen, der zunächst das Ereignis erzählt - Auszug aus Ägypten -, und der dann das Fest zum Gedenken an dieses Ereignis - Pessach - beschreibt und dessen Durchführung durch Bestimmungen regelt. Gerade diese Übereinstimmung von zeitlicher Sequentialität im Erzählten mit der Abfolge des Erzählens wird aber vermieden, statt dessen findet eine Überblendung von Ereignis und Ritual statt. Zu beiden gehört das Schlachten des Lammes als konstitutiver Bestandteil.

Wie in einem Mikrokosmos findet in diesen vier Kapiteln des Exodusbuchs das statt, was als literarisches Grundprinzip der Tora, des Pentateuch, gelten kann: Erzählung und Weisung sind auf einzigartige Weise miteinander verschränkt. Die Tora ist sowohl Erzählwerk als auch Gesetzessammlung. Dabei hängen Gesetz bzw. Weisung5 und Erzählung nicht nur stilistisch-literarisch, sondern auch inhaltlich eng zusammen. Die Grundlage der Weisung bzw. die Bedingung der Möglichkeit tora-gemäßen Handelns liegt in der Befreiungserfahrung, die Israel durch seinen Gott JHWH im Auszug aus Ägypten gemacht hat. Diese Erfahrung wird als Horizont für das Halten der Gebote und Gesetze verstanden. ${ }^{6}$

Im Schlachten des Pessach-Lamms findet diese Entsprechung von konstitutiver Erfahrung und tora-gemäßem Handeln einen ritualisierten Ausdruck. So wie die erste Generation bei ihrem Auszug aus Ägypten ein »fehlerfreies, männliches, einjähriges Lamm« (Ex I2,5) schlachtet, so sollen alle weiteren Generationen ebenfalls ein Lamm schlachten, um des Ereignisses zu gedenken. Im Rahmen der Pessach-

3 | I. Müllner: Pessach.

4 | G. Steins: Gedenken, S. 90.

5 | Das hebräische (tōrāh) meint zunächst die Einzelweisung, wird dann zum Begriff für Lebensweisung insgesamt und schließlich zum metatextuellen Begriff für die damit bezeichneten fünf Bücher Genesis, Exodus, Levitikus, Numeri und Deuteronomium, die in der Perserzeit (ausgehendes 6. bis Mitte des 4. Jh. v.u.Z.) zu einem Korpus zusam-

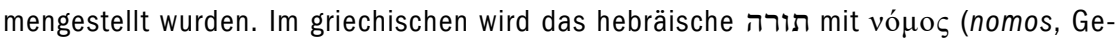
setz) wiedergegeben, was über das lateinische lex zu einer Verengung des Begriffs auf den gesetzlichen Aspekt geführt hat. Demgegenüber soll der Begriff "Weisung" im deutschen an die Weite des hebräischen Tora-Begriffs anknüpfen.

6 | Vgl. F. Crüsemann: Bewahrung. 
erzählung werden noch weitere Opferarten geboten und mit dem - aus der Perspektive der erzählten Welt - zukünftigen Leben im Land in Verbindung gebracht.

${ }^{11}$ Wenn dich nun der HERR ins Land der Kanaaniter gebracht hat, wie er dir und deinen Vätern geschworen hat, und es dir gegeben hat, ${ }^{12}$ so sollst du dem HERRN alles aussondern, was zuerst den Mutterschoß durchbricht. Auch alle männliche Erstgeburt des Viehs, die dir zuteil wird, gehört dem HERRN. ${ }^{13}$ Die Erstgeburt des Esels sollst du mit einem Schaf auslösen; wenn du sie aber nicht auslöst, so brich ihr das Genick. Aber alle Erstgeburt beim Menschen unter deinen Söhnen sollst du auslösen.

${ }^{14}$ Und wenn dich künftig dein Sohn fragen wird: 'Was ist das?, sollst du ihm sagen: ,Der HERR hat uns mit mächtiger Hand aus Ägypten, aus dem Sklavenhaus, geführt. ${ }^{15}$ Denn als der Pharao sich hartnäckig weigerte, uns ziehen zu lassen, erschlug der HERR alle Erstgeburt in Ägypten, von der Erstgeburt des Menschen bis zur Erstgeburt des Viehs. Darum opfere ich dem HERRN alles Männliche, das zuerst den Mutterschoß durchbricht, aber die Erstgeburt meiner Söhne löse ich aus. ${ }^{16}$ Das soll dir ein Zeichen auf deiner Hand und ein Merkzeichen zwischen deinen Augen sein; denn der HERR hat uns mit mächtiger Hand aus Ägypten geführt.‘' (Ex 13,11-16)

Ebenso wie der Tod des Pessachlamms wird auch das Erstgeburtsopfer in ein Korrespondenzverhältnis mit den Vorgängen in der menschlichen Gesellschaft gebracht. Die Tötung der ägyptischen Erstgeborenen als ultima ratio, als zehnte Plage in der Auseinandersetzung zwischen den beiden göttlichen Mächten JHWH und Pharao, steht in einem Verhältnis sowohl zur Schlachtung des Pessachlamms als auch zur Opferung der Erstgeborenen. Das Blut des Lamms an den Türpfosten soll die Israelitinnen und Israeliten schützen. Gleichzeitig entspricht die Schlachtung des Lammes in jedem Haus der Israelit_innen der Tötung der Erstgeborenen von Vieh und Mensch bei den Ägypter_innen. Ebenso wird ein Entsprechungsverhältnis zwischen der Tötung der Erstgeborenen in Ägypten und der Auslösung der Erstgeborenen in Israel hergestellt. Diese Einbindung einer Opferpraxis in ein Narrativ stellt aber noch ein weiteres Entsprechungsverhältnis als das zwischen dem Ereignis und dem Ritual her, nämlich das zwischen Menschen und Tieren.

\section{Biblischer Text und Rituelle Praxis}

Die Praxis des Tieropfers ist in der Antike gängig, wie die Quellen sowohl der griechisch-römischen als auch der altorientalischen Kulturen breit belegen.7 Das Opfer gehört zu den Ritualen, in denen Menschen sich dem Göttlichen nähern, mit diesem Kontakt herstellen und ihre Verehrung zum Ausdruck bringen. Während gegenwärtige, christlich geprägte Vorstellungen von Religion sehr stark

7 | S. ausführlich M.-Z.Petropoulou: Sacrifice. 
mit dem »Glauben«, also mit einem mentalen relationalen Akt in Verbindung gebracht werden, sind antike Religionen viel stärker von ihren Praktiken her bestimmt. Die Religion kommt in der Verehrung nicht einfach zum Ausdruck, sondern die Verehrungspraxis konstituiert Religiosität. Dementsprechend sind die biblischen Regelungen zu angemessener ritueller Praxis keine Frage von Äußerlichkeit im Verhältnis zu irgendeiner zu vermutenden Innerlichkeit, sondern sind Formierungen der Religion selbst. Diese gehört wesentlich zur Identität des biblischen Israel und wird deshalb insbesondere in der exilisch-nachexilischen Epoche intensiv verhandelt.

Dabei darf kein einliniges Verhältnis von biblischem Text und ritueller Praxis vorausgesetzt werden. Inwieweit die Rechtstexte kultischer Praxis entsprechen, ist immer wieder Gegenstand von Forschungsdebatten. ${ }^{8}$ Zumindest aber wird darauf hinzuweisen sein, dass der hohe Regelungsbedarf im kultischen Bereich hinsichtlich der Angemessenheit von Ort, beteiligten Akteur_innen und zu vollziehenden Handlungen darauf hinweist, dass durchaus andere als die im überlieferten Bibeltext gebotenen rituellen Praktiken gängig waren. Auch das Einbeziehen narrativer Texte zu rituellen Themen weist in diese Richtung - manche Praktiken sind zwar in den Augen der Träger_innengruppen der Texte dem JHWH-Kult unangemessen (z.B. die Opferung von säugenden Kühen); sie werden aber als Praxis erzählt (I Sam 6). Festzuhalten bleibt aber, dass es sich bei den Ritualtexten der Bibel eben um Texte handelt und nicht um rituelle Praxis. Religionswissenschaftler wie Jonathan Z. Smith sehen deshalb die in der Tora beschriebenen Rituale nicht als Gegenstand ritualwissenschaftlicher Forschung an:

"[...] we don't have ritual texts in the bible. We have very poor ethnographic descriptions. You cannot perform a single biblical ritual on the basis of what is given to you in the text. If you can't perform it, then by definition it is not a ritual. The biblical texts are scattered, theoretical reconstructions of what may have happened." ${ }^{9}$

Nicht nur aus literaturwissenschaftlicher, sondern auch aus exegetischer Sicht ist die Einengung der Fragestellung auf den Praxisgehalt der Weisungstexte unzulänglich. Weder die biblischen noch andere altorientalische Rechtstexte lassen sich als Fenster zur Rechtspraxis verstehen; sie sind Literatur. ${ }^{\text {IO }}$ Deshalb wird man sich den biblischen Opferbestimmungen ebenso wie den Erzählungen über Opfer zwar auch mit religionswissenschaftlichen Theorien annähern können, diese aber in eine philologische Untersuchung zu integrieren haben. Diese Texte

8 S. mit einer klaren Positionierung aufseiten der Literarizität der Rechtstexte H. Liss: Kanon; dort auch weitere Literatur.

9 | Jonathan Z. Smith in einem Gespräch zit. n. Hamerton-Kelly: Violent Origins, S. 210.

10 | H. Liss: Kanon. 
wollen zuallererst gelesen und ausgelegt, nicht abbildhaft in Handlung umgesetzt werden. ${ }^{\text {II }}$

Hier kann es deshalb auch nicht in erster Linie um eine Diskussion theoretischer Konzepte gehen, deren Ziel es ist, Opferpraxis begrifflich zu fassen. ${ }^{22}$ Dennoch wird es hilfreich sein, zentrale Aspekte dieser Diskussion aufzugreifen und mit der Frage nach der Rolle von Geschlecht in den biblischen Ritualtexten in Zusammenhang zu bringen. Dabei sind vor allem zwei Deutungslinien zu berücksichtigen: eine pragmatische Deutung, die sich auf die Haltung von Nutztieren bezieht, und eine symbolistische, in der die Opferrituale in ihrer Korrespondenz mit sozialen Ordnungen und Vollzügen gedeutet werden. ${ }^{\mathrm{I3}}$

\section{Opferarten und ihre geschlechtlichen Spezifika}

In den alttestamentlichen Texten werden unterschiedliche Opferarten benannt und beschrieben. Diese werden in den Regelungsbestimmungen für Rituale, wie sie in der Tora zu finden sind, auch hinsichtlich der dabei zu verwendenden Tiere näher bestimmt.

Die Bezeichnung der Opferriten unterliegt keiner klaren Systematik. Hier können etwa der Anlass (חָטָאת, chatā't, Sündopfer; oder auch die Art und Weise der Opferung (עוֹלָה, 'ōlāh, Brandopfer; קִטרוֹרה, qetōrāh, Räucheropfer) namengebend sein. ${ }^{\mathrm{I}}{ }^{2}$ Die Regelungen einzelner Opferarten werden hinsichtlich ihres Anlasses, des sozialen Status der Darbringenden, des Ortes des Opfers, der dabei zu vollziehenden Handlungen, des Zugangs zur Opfermahlzeit und der dabei zu verwendenden Tiere geregelt. Dabei spielen neben der Spezies auch das Geschlecht, das Alter und der körperliche Zustand der Tiere eine Rolle.

In Lev I- 5 werden verschiedene Arten des Opfers benannt und ihre Ausführungen beschrieben; in Lev 6-Io werden weitere Opferbestimmungen, vor allem für die Priester, ausgeführt. Für diese Ausführungen sind vor allem Lev I-5 relevant. Deshalb soll im Folgenden eine kurze Übersicht über die mit den Tieropfern verbundenen Regelungen gegeben werden. Sie werden paradigmatisch behandelt,

11 | Vgl. F. Bark: Heiligtum, zu den Texten, die den Bau der Stiftshütte beschreiben. Sie geht von der Beobachtung aus, dass es sich hier nicht um Baupläne handelt, die auf eine Umsetzung in Bautätigkeit zielen, sondern um Texte, die im Prozess der Lektüre "ein Heiligtum im Kopf der Leser“ entstehen Iassen sollen. S.a. H. Liss: Kanon, pass.

12 | S. dazu ausführlich J. Klawans: Purity, S. 3-10; 17-48.

13 | Beide Deutungslinien werden auch von N. Ruane: Sacrifice, herangezogen. Dabei kann die erste sich eher im Horizont der von J.Z. Smith: Domestication, stark gemachten These verstehen, die zweite eher auf die Arbeiten von Mary Douglas, v.a. M. Douglas: Purity, und Douglas: Leviticus, rekurrieren.

14 | U. Dahm: Art.: Opfer; ausführlicher Th. Hieke: Levitikus, S. 80-104. 


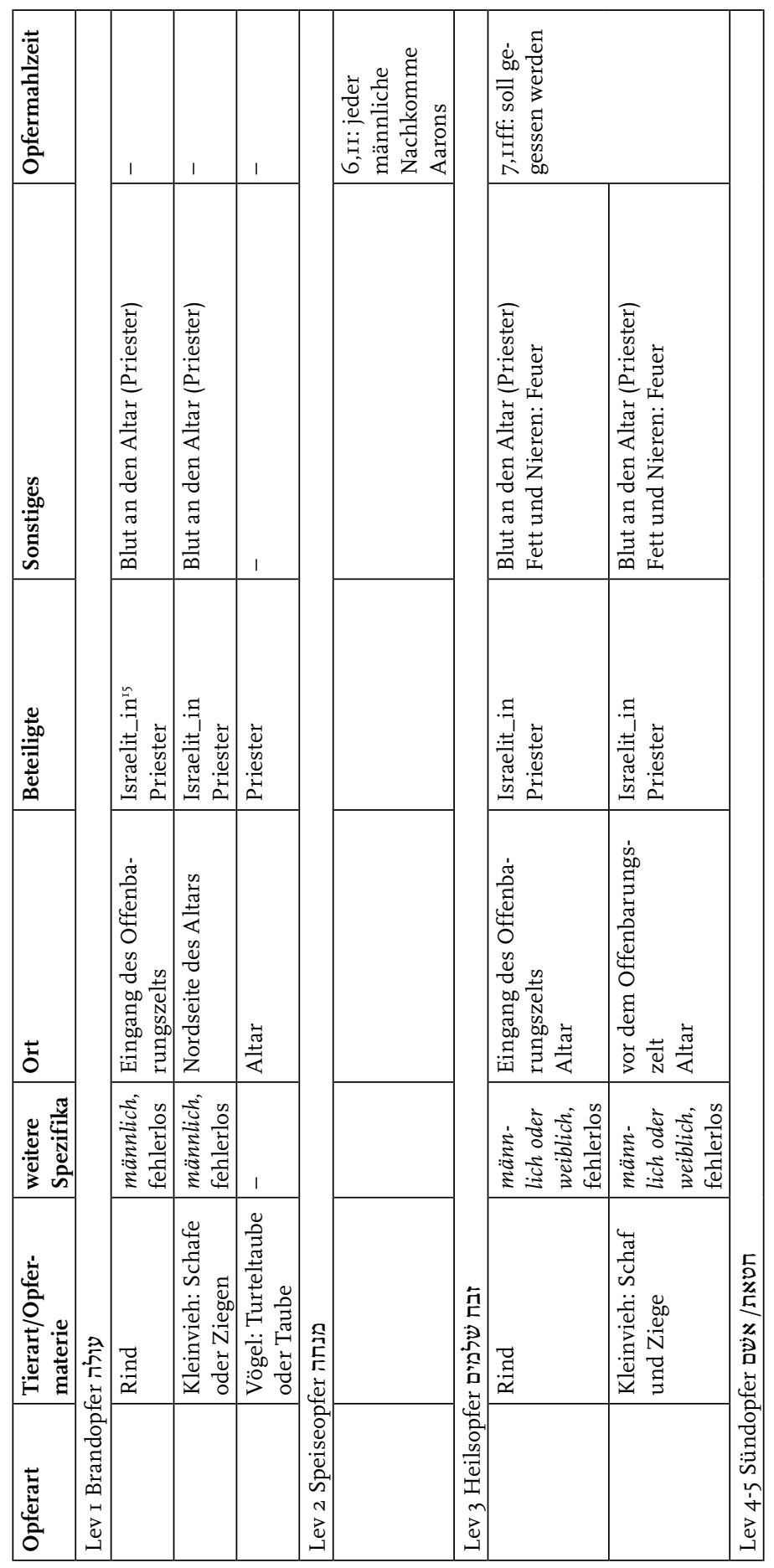




\begin{tabular}{|c|c|c|c|c|c|c|c|c|}
\hline \multicolumn{5}{|l|}{ 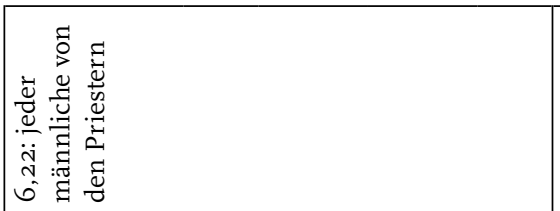 } & \multicolumn{4}{|l|}{ 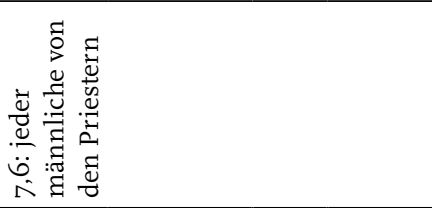 } \\
\hline 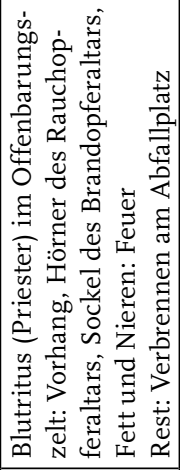 & 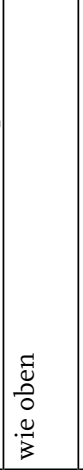 & 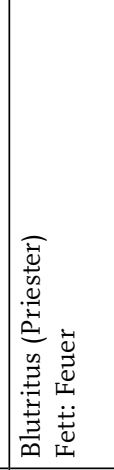 & 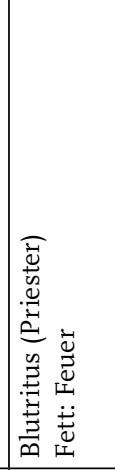 & 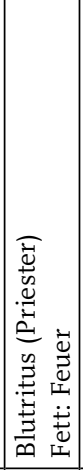 & & & & \\
\hline 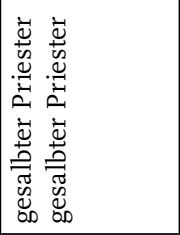 & 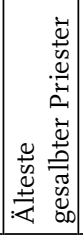 & 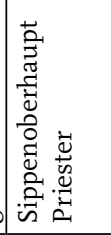 & 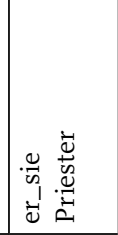 & 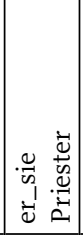 & & & & \\
\hline 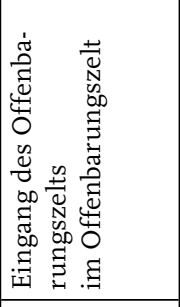 & 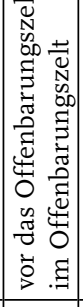 & 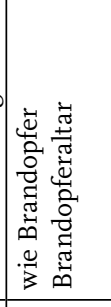 & 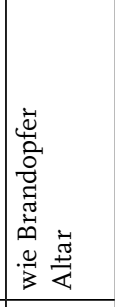 & 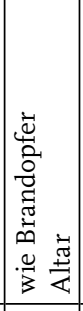 & & & & \\
\hline 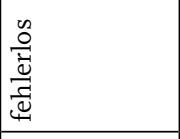 & & 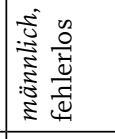 & 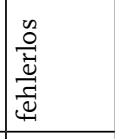 & 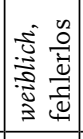 & 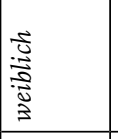 & & & 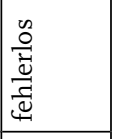 \\
\hline 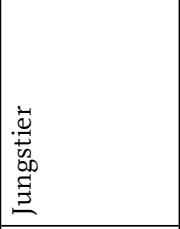 & 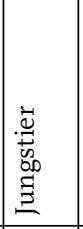 & 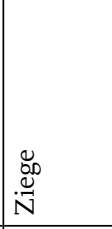 & 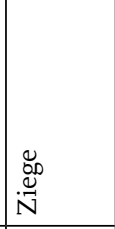 & 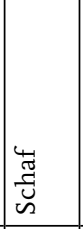 & 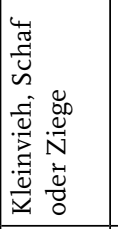 & 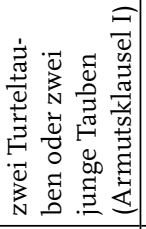 & 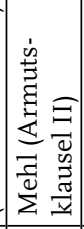 & 莕 \\
\hline 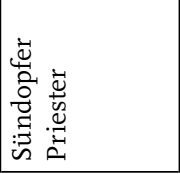 & 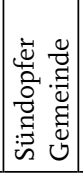 & 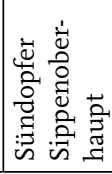 & 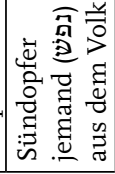 & & 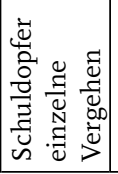 & & & 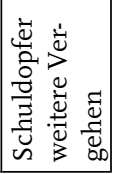 \\
\hline
\end{tabular}


da eine vollständige Übersicht über die für die Opfer relevanten Textstellen den Rahmen dieser Ausführungen gänzlich sprengen würde.

Das Geschlecht spielt bei der Bestimmung der Opfertiere neben der Spezies und der Feststellung der Fehlerlosigkeit eine zentrale Rolle. Explizit erwähnt wird das Geschlecht in Lev I-5 bei allen Opferarten, das Fehlen einer geschlechtlichen Markierung bildet die Ausnahme. Nur die Vögel des Brandopfers und des Schuldopfers bleiben geschlechtlich unbestimmt, das Geschlecht der Säugetiere wird genannt. Dabei ist auch die Formulierung »männlich oder weiblich« (אם־זכר אם־נקבה) als Berücksichtigung des Geschlechts aufzufassen, nur, dass es in diesem Fall (Heilsopfer) möglich ist, sowohl männliche als auch weibliche Tiere zu opfern. ${ }^{\mathrm{I}}{ }^{6}$

Die im Alten Testament am häufigsten genannte Opferart ist das Brandopfer (עולה, 'ólāh $)^{17}$. Es steht für die vollständige Hingabe an das Göttliche. Das Kennzeichen der עולה ist, dass dieses Opfer zur Gänze verbrannt wird, ohne dass ein Teil des Tieres (mit Ausnahme des Fells für die Priester, Lev 7,8) zur weiteren Verwendung übrig bleibt. In den rechtlichen Regelungen besteht Eindeutigkeit dahingehend, dass die עולה immer männlich zu sein hat, wenn es sich um ein Rind, ein Schaf oder eine Ziege handelt. ${ }^{18}$ Außerhalb der rechtlichen Bestimmungen allerdings gibt es durchaus die Opferung weiblicher Tiere als עולה. Narrativ entfaltet wird z.B. die Opferung von säugenden Kühen anlässlich der Überführung des Schreins vom philistäischen in israelitisches Gebiet in I Sam 6,7-I4. Die Kühe, die den Schrein ohne menschliches Zutun und gegen ihre natürliche Bewegung weg von den Kälbern bis nach Bet-Schemesch gezogen haben, werden an-

15 | Ob grammatikalisch maskuline Formulierungen in Rechtstexten tatsächlich Männer meinen oder aber geschlechtsneutral zu verstehen sind, ist oft nicht festzustellen. S. dazu I. Müllner: Konstruktionen, S. 119-127.

16 | Allerdings ist das Geschlecht eines Tieres von der Bezeichnung her nicht immer eindeutig zu bestimmen. Gerade die Gattungsbezeichnungen von Tieren sind auch im Hebräischen häufig geschlechtlich mehrdeutig (man denke an das deutsche Wort "Katze»). Die rechtlichen Regelungen machen die geschlechtlichen Bestimmungen zwar häufig explizit, in anderen Texten ist eine solche Klarheit nicht immer gegeben. Insbesondere dann, wenn grammatisches und natürliches Geschlecht nicht übereinstimmen (müssen), ist eine solche Eindeutigkeit schwierig herzustellen. N. Ruane: Sacrifice, S. 43-45.

17 | Die etymologische Herleitung des Begriffs ist unklar. Wahrscheinlich leitet er sich vom Verb עלה, 'ālāh, aufsteigen ab. Das Brandopfer lässt die Opfermaterie in Gänze in Rauch aufsteigen, der Priester steigt zur Opferplattform hinauf, das Opfer zum Altar. Vgl. Th. Hieke: Levitikus, S. 83. Andere deutsche Übersetzungsmöglichkeiten sind "Ganzopfer" oder "Darhöhung" (Martin Buber). Zum Brandopfer in Gen 22 siehe auch den Beitrag von Y.S. Thöne in diesem Band.

18 Dass die Tauben geschlechtlich unbestimmt bleiben, hat wahrscheinlich praktische Gründe. Th. Hieke: Levitikus, S. 185. Eine Erklärung dafür muss aber spekulativ bleiben. 
lässlich der Ankunft dieses Kultgegenstands geopfert. ${ }^{\text {I9 }}$ Allerdings handelt es sich bei allen hier infrage kommenden Fällen ${ }^{20}$ um spontane Rituale, die sich jenseits der räumlichen und zeitlichen Ordnungen des Jerusalemer Tempels befinden, für die bei der עולה eine Festlegung auf männliche Opfertiere gilt. Da es im Rahmen des Brandopfers kein gemeinsames Opfermahl gibt, sind die am Ritual Beteiligten jene Menschen, die mit der Schlachtung, dem Blutritus und dem Verbrennen der Opfermaterie zu tun haben. Die beteiligten Priester sind männlich, der (ādām, Mensch), der laut Lev 5,2 das Opfertier heranbringt, kann geschlechtsneutral oder männlich aufgefasst werden. אדם bedeutet Mensch oder auch Mann ein Kennzeichen androzentrischer Sprache, das eine Bestimmung des Geschlechts unmöglich macht, wenn nicht noch andere Hinweise gegeben werden.

"Es spielt nicht nur das Geschlecht des/der Opfernden keine Rolle, auch wird nicht angegeben, ob er/sie einer bestimmten Gruppe, Schicht oder sozialen Klasse angehören muss. Diese grundsätzliche Beobachtung kollidiert mit dem weiteren Verlauf des Textes, wo - zumindest grammatikalisch - immer von einem männlichen Opfernden die Rede ist. [...] Solange keine andere Evidenz vorliegt, muss grundsätzlich davon ausgegangen werden, dass Frauen wie Männer an derartigen Darbringungen beteiligt waren «. ${ }^{21}$

Festzuhalten bleibt aber, dass eine geschlechtliche Spezifizierung in diesem Text offenbar nicht von Interesse ist. ${ }^{22}$

Das Heilsopfer (זבח שלמים, zaevach schelāmìm) hingegen benennt ausdrücklich die Möglichkeit, männliche oder weibliche Opfertiere darzubringen. Eine unterschiedliche Behandlung der Tiere des einen oder anderen Geschlechts wird dabei nicht vorgenommen. Mit den beiden Adjektiven זכר (zāchār, männlich) und נקבה ( $n^{e}$ qevāh, weiblich) wird im Hebräischen das benannt, was wir heute wohl als biologisches Geschlecht (sex) bezeichnen würden. ${ }^{23}$ Als Opfertiere kommen Rinder, Schafe oder Ziegen in Betracht. Wie beim Brandopfer wird auch beim Heilsopfer der fehlerlose Zustand des Opfertieres betont. Die Aspekte der Fehlerlosigkeit werden in Lev 22,I7-25 geregelt. Es soll ausgeschlossen werden, dass das Opfertier

19 | Vgl. zu dieser Erzählung I. Müllner: Samuelbücher, pass.

20 N. Ruane: Sacrifice, S. 47-49, nennt außerdem noch die Opferung von Jiftachs Tochter (Ri 11).

21 Th. Hieke: Levitikus, S. 161. S.a. ausführlich und bereits zu Beginn der 90er Jahre G. Braulik, Frauen. N. Ruane: Sacrifice, S. 18-39, diskutiert die verschiedenen Rollen beim Opfer unter geschlechtsspezifischer Perspektive. Sie ist an dieser Stelle zurückhaltender, was die geschlechtsoffene Interpretation androzentrischer maskuliner Begrifflichkeit betrifft.

22 | S. dazu I. Müllner: Konstruktionen, bes. S. 119-127. Vgl. K. Finsterbusch: Frauen; M. George: Masculinity.

23 | Zur Unterscheidung zwischen sex und gender und zur Diskussion der Begriffe in der Genderforschung s. einführend C. v. Braun, I. Stephan: Gender@Wissen. 
irgendeinen angeborenen oder etwa durch Verletzung entstandenen körperlichen Schaden hat. Damit will das priesterliche Gesetz wohl ausschließen, dass man Tiere, die nicht (mehr) viel wert sind, im Rahmen der Opferhandlungen entsorgt. ${ }^{24}$

Stärkere soziale Differenzierungen sowohl auf der menschlichen als auch auf der tierlichen Seite werden in den Regelungen der Sündopfer (חטאת, chatä't) vorgenommen, die Lev 4 entfaltet. Je nachdem welchen sozialen Status die Person hat, der ein Vergehen zur Last gelegt wird, die also einer Ent-schuldigung bedarf, wird aufseiten der geopferten Tiere differenziert. Auf der Seite der Menschen ist zwischen Kollektiv- und Individualsubjekten zu unterscheiden. Als Kollektivsubjekt wird die gesamte Gemeinschaft Israel (כל־עדת ישראל, kol-'edat jisra'el) benannt, die in der Opferhandlung durch ihre Ältesten vertreten wird. Wenn man von der Opfermaterie auf die Relevanz der Handlung Rückschlüsse ziehen darf, dann ist das Vergehen der gesamten Gemeinde vergleichbar mit dem des einzelnen Priesters. In beiden Fällen wird ein junger Stier, also ein sehr wertvolles Tier, ${ }^{25}$ geopfert. Der Rang dieser Opferhandlung wird zusätzlich dadurch betont, dass das gesamte Tier der Gottheit dargebracht wird.

Wenn allerdings ein Sippenoberhaupt oder aber jemand (שפנ) aus dem Volk die Sünde begangen hat, dann findet ein grundsätzlich anderer Opferritus statt. Dieses Sündopfer wird vollständig außerhalb des heiligsten Raums belassen, das Blut wird nicht innerhalb des Zelts auf den Altar gesprengt wie bei den bislang behandelten Opferriten, sondern auf dem außerhalb des Allerheiligsten liegenden Brandopferaltar aufgebracht. Außerdem wird das Opfertier nicht vollständig verbrannt, sondern sein Fleisch wird von den Priestern gegessen. Auch hinsichtlich von Spezies und Geschlecht unterscheiden sich die Sündopferriten. Bei dem Sippenoberhaupt oder bei einer Person aus dem Volk als Opfernden werden Schafe oder Ziegen verwendet, im Fall des Sippenoberhaupts ein männliches, im Fall einer anderen Person ein weibliches Tier. Auch für weitere Vergehen, die ein einzelner Israelit oder eine einzelne Israelitin begehen kann, und die in Lev 5 entfaltet werden, sind weibliche Tiere, Schafe oder Ziegen, ${ }^{26}$ als Opfer vorgesehen.

Es liegt nahe, hier sowohl auf der Seite der Menschen als auch auf jener der Tiere ein Gefälle in der sozialen Ordnung wahrzunehmen. Das weibliche Tier wird von demjenigen Menschen, einer nicht näher spezifizierten Person aus der Gemeinde der Israelit_innen, dargebracht, der oder die sozial keinen herausgehobenen Rang einnimmt. Sowohl der einzelne Priester als auch die Gemeinschaft insgesamt oder auch das Sippenoberhaupt sind sozial als höherrangig klassifiziert; das ihnen zugewiesene Opfertier ist männlich.

24 | Vgl. Th. Hieke: Levitikus, S. $162 f$.

25 | Ebd., S. 162.

26 | Die Übersetzung der Lutherbibel mit "Schaf- oder Ziegenmutter", die auch in der Revision von 1984 perpetuiert wird, ist nicht nur dem Text von Lev 5,6 nicht gemäß - dort steht einfach נקבה sie läuft auch der Vermeidung des Mütterlichen im Bereich des Opfers (s.u.) gänzlich zuwider. 
Dieses Korrespondenzverhältnis zwischen dem Geschlecht des Opfertiers und dem sozialen Status des oder der Opfernden bzw. dem Stellenwert der Opferart wird einer der Schlüssel sein, mit denen im nächsten Abschnitt eine Annäherung an die Deutung dieses Phänomens gesucht werden soll.

\section{Opfer, Reinheit, Heiligkeit und die Ordnungen des Raums}

Opfertiere sind also in den Opferbestimmungen der Tora - hier paradigmatisch entfaltet an jenen des Buchs Levitikus - geschlechtlich bestimmt. Dabei fällt auf, dass männliche Tiere bevorzugt werden. Beim wohl höchstrangigen Opfer, dem Brandopfer (עולה) werden ausschließlich männliche Tiere verwendet. Die Differenzierungen der unterschiedlichen Sündopferriten haben gezeigt, dass in diesem Kontext männliche Tiere höherrangige Personen repräsentieren. ${ }^{27}$

Es stellt sich die Frage, wie diese Zuweisung von Geschlecht verstanden und auch, wie sie in eine weitere Theorie von Geschlecht im Rahmen der regulativen Bestimmungen der Tora ${ }^{28}$ integriert werden kann. Dazu ist es sinnvoll, den Horizont der Fragestellung zu erweitern. Wie Jonathan Klawans überzeugend gezeigt hat, werden in der Literatur meist zwei Bereiche, die für die priesterlichen Regulative zentrale sind, getrennt voneinander behandelt: Opferregelungen und Reinheitsvorschriften. Allerdings ermöglicht eine Zusammenschau der beiden, die von den Texten her auf der Hand zu liegen scheint, ein vertieftes Verständnis sowohl von Reinheit als auch von Opfer und außerdem einen umfassenderen Zugang zum Heiligkeitskonzept der priesterlichen Literatur. ${ }^{29}$

Der Zusammenhang zwischen Reinheit und Opfer ist komplex. Die beiden Bereiche - Opferrituale und Reinheitsvorschriften - überschneiden sich in einzelnen Bestimmungen. So wird etwa der Abschluss der Phase der Kultunfähigkeit der Wöchnerin, wie sie in Lev I2 entfaltet werden, durch ein Opfer zelebriert. Ähnlich werden die Reinigungsriten von sexuellen Ausflüssen bei Männern und Frauen mit einer Opferhandlung verbunden (Lev I5). Ein sehr intensives Korrespondenzverhältnis von Reinheit und Opfer wird in Num ig entwickelt, wo der außergewöhnliche Ritus der Schlachtung der roten Kuh zur Herstellung des Rei-

27 | Der Zusammenhang zwischen Opferndem und Opfer ist nicht von außen an das Geschehen herangetragen, sondern wird insbesondere durch eine rituelle Handlung, die die Opferbestimmungen durchzieht, immer wieder aktualisiert. Der Ritus der Handaufstemmung stellt den "Zusammenhang zwischen Mensch und Tier, zwischen der Opfermaterie und der menschlichen Person, um deren Gottesbeziehung es geht", her. Th. Hieke: Levitikus, S. 169. Die enge Verbindung zwischen den Opfernden und der Opfermatierie wird also performativ inszeniert in eben diesem Ritus der Handaufstemmung.

28 | S. dazu ausführlicher I. Müllner: Konstruktionen.

29 | J. Klawans: Purity, pass. 
nigungswassers beschrieben wird. ${ }^{30}$ Hier ist das Sündopfer (חטאת) die auch materielle Ermöglichung eines Reinigungsrituals. Es gibt also mehrere Stellen, an denen einzelne Opferrituale und Reinigung miteinander verbunden werden.

Opferrituale und Reinheitsvorstellungen korrespondieren aber auch grundsätzlicher, insofern als die Opferung unreiner Tiere oder anderen Materials ebenso undenkbar wäre wie das Betreten des Heiligtums durch Menschen, die sich im (vorübergehenden) Zustand kultischer Unreinheit befinden. Rituelle Reinigung ist Teil des Opferprozesses, wenn man diesen nicht auf den Akt des Schlachtens reduziert. ${ }^{\text {I }}$

Die Verbindung zwischen den beiden Bereichen Opfer und Reinheit geht also über ihre partiellen Schnittmengen hinaus und findet sich in einem übergreifenden Prinzip, das beide verbindet: der Heiligkeit.

"One organizing principle is the concern with imitating God. Another organizing principle is the concern with attracting and maintaining the presence of God within the community. By focusing these two concerns, we will be able to analyze the two sets of ritual structures - sacrifice and defilement - in tandem. ${ }^{32}$

Beide Anliegen - die imitatio Dei ebenso wie die Präsenz des Göttlichen in der Gemeinschaft - werden im Begriff der Heiligkeit gefasst, der in den biblischen Texten entwickelt wird. Damit wird deutlich, dass beide Bereiche - Opfer und Reinheit - Funktionen eines zentralen Konzepts, nämlich der Heiligkeit sind. Dieses zentrale Konzept wiederum ist in seiner Grundstruktur ein räumliches, bezogen auf das Heiligtum.

"The status of impurity is the opposite of the status of purity. Both conditions are related only to the sanctuary and do not describe a physical condition as such. The sanctuary requires the status of purity. [...]

The starting point of the whole idea is God's holiness; he is qādôš. Unlike the created world he is never subject to the categories impure ‘ tāmē or 'pure tāhôr. Both areas the holy and the common - are strictly separated. And it is only with the concept of the tabernacle/the tent of meeting (miškān; 'ōhel mô 'ēd) that the area of holiness exceeds its limits. The categories impure ' tāmē and 'pure tāhôr had to be introduced to enable the holy to meet the common. Within this system of spatial categories, holiness cannot spread diffusely into common spaces, because this would mean the annuling of the whole system: holy space requires common space as its boundaries." ${ }^{33}$

30 | Diese für das hier verhandelte Thema relevante Passage kann aus Raumgründen im vorliegenden Aufsatz nicht verhandelt werden. Ausführlich diskutiert N. Ruane: Sacrifice, S. 106-147, dieses Ritual.

31 | J. Klawans: Purity, pass.

32 Ebd., S. 48.

33 | H. Liss: Mice, S. $207 f$. 
So entsteht eine konzentrische Raumstruktur, in der das Allerheiligste den Mittelpunkt bildet und die durch die Raumpraktiken von Menschen und Tieren immer wieder hergestellt wird. Mit Ellen van Wolde lässt sich diese Raumordnung der Heiligkeit schematisch darstellen:
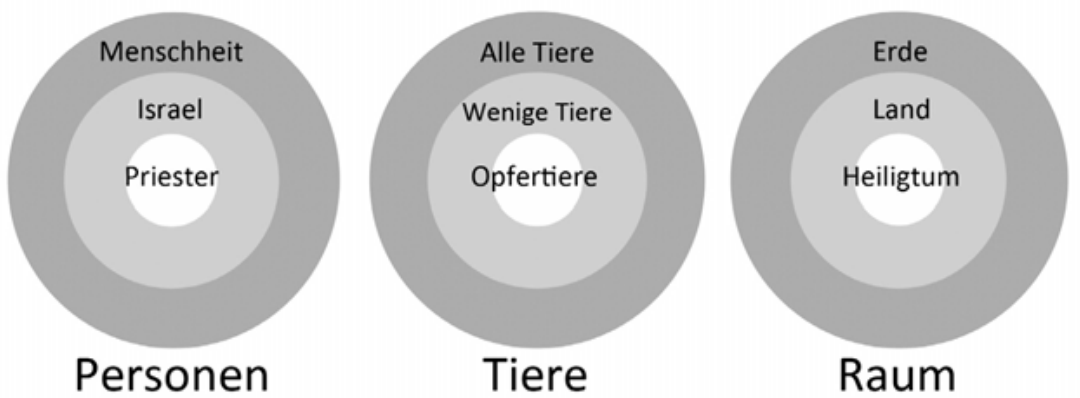

Abb. 1: Räumliche Ordnung von Heiligkeit ${ }^{34}$

Das Zusammendenken der Opferrituale und des Reinheitssystems ermöglicht auch, auf Arbeiten zurückzugreifen, die sich mit der symbolischen Ordnung des Reinheitssystems befassen. Die berühmt gewordene Formulierung von Mary Douglas »Where there is dirt there is a system « ${ }^{35}$ steht für einen Ansatz, der die Verbannung der Reinheitsvorstellungen ins Chaotisch-Primitive überwindet und die soziale Bedeutung eines solchen Ordnungssystems deutlich macht. Hier ist der Ansatzpunkt, um auch die Opferpraktiken, im Fall der vorliegenden Überlegungen ihre Konstruktion von Geschlecht als Symbolsysteme, zu reflektieren. Die Parameter Spezies, Geschlecht, Alter und Un-/Versehrtheit bilden Aspekte eines Symbolsystems aus, das in Korrelation mit der menschlichen Sozialstruktur zu bringen ist. Dabei spielen Wertigkeiten eine Rolle, sind aber nicht das einzige relevante Moment. Ein solcher symbolistischer Zugang zur Deutung der Opfervorstellungen stellt nicht die Gewalt ins Zentrum seiner theoretischen Herangehensweise, sondern die sozialen Funktionen der Opfer. Auch wenn klar sein muss, dass Opferrituale auch nur einer einzigen Gesellschaft ein zu komplexes System bilden, als dass sie mit einem einzelnen theoretischen Zugriff umfasst werden könnten. ${ }^{36}$

Das Geschlecht der Opfertiere ist damit Teil eines Menschen, Tiere und das Göttliche umfassenden geschlechtlichen Symbolsystems, das durch komplexe Handlungszusammenhänge, zu denen auch die Opferrituale gehören, immer

34 | E.J. v. Wolde: Reframing, S. 208, im Anschluss an Jacob Milgrom.

35 | M. Douglas: Purity, S. 44.

36 | Das betonen beinahe alle Theoretikerlnnen, die sich mit dem Begriff des Opfers beschäftigen, z.B. J. Milgrom: Leviticus, S. 1-16; 442-443. 
wieder hergestellt wird. Das Männliche - sowohl aufseiten der Menschen als auch aufseiten der Tiere - hat dabei einen weniger restringierten Zugang zum Heiligen, ins Zentrum des Allerheiligsten gelangen ausschließlich männliche Wesen.

"The exclusion of the female animal from the 'ôlâ sacrifice, arguably the most important and prestigious offering, also reflects the exclusion of the female from the highest levels of sanctitiy and most prestigious aspects of cultic participation." ${ }^{37}$

Die symbolische Höherwertung des Männlichen trifft auf ein zweites Erklärungsmuster der Bevorzugung männlicher Opfertiere, das diesem symbolistischen auf den ersten Blick widerspricht: der relativen Nutzlosigkeit der männlichen Tiere in der Herde.

\section{Haustierhaltung, Zucht UNd ÜBerLeben}

Dieses Erklärungsmuster setzt bei der These an, dass das Opfer mit der Wirtschaftsform der Haustierhaltung in Zusammenhang zu bringen ist. Die soziale Verortung des Opfers in der Viehzüchtergesellschaft geht auf Jonathan Z. Smith zurück, der sich damit von älteren Theorien absetzt, wonach das Opfer eine Funktion der Jägergesellschaft wäre. ${ }^{38}$

"I know of no unambiguous instance of animal sacrifice that is not of a domesticated animal. [...] I know of no unambiguous instance of animal sacrifice performed by hunters. Animal sacrifice appears to be, universally, the ritual killing of a domesticated animal by agrarians or pastoralist societies. ${ }^{39}$

Damit steht die Opferung als Ritual in einem ökonomischen Kontext, in dem es um Zucht und Haltung von Haustieren geht. Auch in den biblischen Regelungen werden ausschließlich Opfertiere erwähnt, die im Israel der Antike als Haustiere gehalten wurden - das Beispiel der Taube bzw. Turteltaube als einzigem als Haustier bekannten Vogel und eben auch als einzigem Vogel, der geopfert wird, macht dies deutlich. Umgekehrt gilt allerdings nicht, dass alle Haustiere auch für Opferungen vorgesehen sind - Equiden z.B. sind keine Opfertiere.

Eine solche Sichtweise ist kein Widerspruch zu dem oben vertretenen Ansatz, die regulativen Texte der Tora nicht primär als sozialgeschichtliche Dokumente, sondern zunächst als literarische Texte zu lesen. Denn auch literarische Texte tragen ein Weltwissen mit sich, das sich unter anderem aus sozialen Erfahrungen speist. Allerdings verbietet der Ansatz beim Text als literarischem einen all-

37 | N. Ruane: Sacrifice, S. 47.

38 | J.Z. Smith: Domestication, im Gegenüber etwa zu W. Burkert: Homo necans.

39 | J.Z. Smith: Domestication, S. 149. 
zu unmittelbaren Zugang zur gelebten Wirklichkeit, eine allzu »enge Text- und Ereigniskorrelation ${ }^{4}{ }^{\circ}$, und gebietet Vorsicht in Bezug auf die Rückschlüsse von den Inhalten des Texts auf das gelebte Leben der Entstehungszeit. ${ }^{4}{ }^{\mathrm{I}}$ Wenn hier also ökonomische und sozialgeschichtliche Kategorien herangezogen werden, so beziehen sich diese im vorliegenden Argumentationszusammenhang immer noch auf die in den Texten entworfene soziale Wirklichkeit.

Die Reflexion über das Geschlecht der Opfertiere führt im Kontext der Haustierhaltung zur Frage nach dem ökonomischen Wert der Tiere. Dieser Wert besteht in der Produktion von Produkten wie Milch, Wolle, Fell und Fleisch. Milch und Wolle, die von lebenden Tieren und daher wiederholt bzw. regelmäßig gewonnen werden können, sind weit nachhaltigere Produkte als Fleisch und Fell, deren Gewinnung den Tod der Tiere voraussetzt. Für die Produktion von Nachkommenschaft sind deutlich mehr weibliche Tiere nötig als männliche; das in der Subsistenzwirtschaft nötige Produkt Milch ist ebenfalls eines, für das weibliche Tiere gebraucht werden. In vielen Herden leben vor allem weibliche und sehr wenige ausgewachsene männliche Tiere - oft sogar nur eines. Männliche Tiere werden deshalb in subsistenzorientierten landwirtschaftlichen Zusammenhängen häufig vor dem Erreichen des Erwachsenenalters geschlachtet, ihr Hauptprodukt ist das Fleisch. In einem System, in dem das Schlachten vorwiegend oder sogar ausschließlich an das ritualisierte Opfer gebunden ist, werden daher vorwiegend männliche Tiere als Opfertiere und damit auch zur Fleischproduktion verwendet.

"[B]y their very nature, sacrificial systems require the deaths of their victims, and because of material concerns related to the husbandry of domesticated animals, male animals make better and therefore more commonly used sacrificial victims. Similarly, females are not often victims in sacrificial practice because of their reproductive value. [...] Thus while being symbolic systems, as well as means of organizing kinship relations and methods of eating meat, ancient sacrificial systems are also systems for culling animals that are no longer useful for the flock or herd." ${ }^{42}$

Symbolischer und ökonomischer Wert müssen nicht in allen Systemen Hand in Hand gehen. Im Fall subsistenzorientierter Landwirtschaft und einem auf das Heiligtum zentrierten Opfersystem gehen die beiden Sichtweisen ein komplementäres Verhältnis ein, das in der Privilegierung der männlichen Opfertiere seinen gemeinsamen Nenner findet.

40 | H. Liss: Kanon, S. 8.

41 | S. z.B. zum komplexen Verhältnis von Profanschlachtung und ritueller Schlachtung im Horizont des Fiktionalitätsparadigmas ebd., S. 27-30.

42 | N. Ruane: Sacrifice, S. 62. 


\section{Mütterliche Materie}

Wenn wir vom Opfersystem als einem Symbolsystem sprechen, dann geht es nicht einfach um dichotomische Wertungen von Männlichkeit und Weiblichkeit: Das geschlechtliche Symbolsystem umfasst weitere Aspekte. Einer davon ist die Bedeutung von Reproduktion, wie sie im Blick auf menschliche und tierliche Mütter und Nachkommen zum Ausdruck kommt. Im Folgenden sollen jene Regelungen in den Blick genommen werden, die von tierlichen Müttern und ihren Nachkommen handeln und von dem, was ich »mütterliche Materie« nennen will, ${ }^{43}$ also Milch und Eier (im Fall der menschlichen Mütter wäre hier auch die Blutung nach der Geburt zu verhandeln).

Im Unterschied $\mathrm{zu}$ anderen altorientalischen Kulturen kennt das biblische Israel keine Opferung von Milch. Als tierliche, flüssige Opfermaterie gilt Blut, die Opferung von Milch wird nicht erwähnt. Damit wird Materie, die spezifisch weiblich und gleichzeitig an die Mutter-Kind-Dyade gebunden ist, vom Opferritual ausgeschlossen. In die gleiche Richtung weisen auch andere Regelungen, die die Milch von Tieren betreffen. Außerdem kennen die biblischen Regelwerke einen Ausschluss von sehr jungen Tieren, die sich noch bei ihrer Mutter aufhalten, von Muttertieren und Jungtieren gemeinsam (Lev 22,27-28) und von Vögeln mit Eiern oder Jungtieren in ihrem Nest (Dtn 22,6-7). Auch das Erstgeburtsopfer geschieht erst nach einer Frist von sieben Tagen, in denen das Jungtier bei seiner Mutter zu bleiben hat (Ex 22,28-29). In die gleiche Richtung weist das Verbot, »das Zicklein in der Milch seiner Mutter zu kochen« (Ex 23,I9; 34,26; Dtn I4,2I), das in der weiteren jüdischen Entwicklung der Kaschrut, der Speisevorschriften, eine wichtige Rolle spielt. Dieses dreifach überlieferte Verbot ist übrigens der einzige Ort der regulativen Texte, an denen Milch eine Rolle spielt. ${ }^{44}$ Allen diesen Texten gemeinsam ist, dass sie die Mutter-Kind-Dyade in den Blick nehmen, die auch in Bezug auf menschliche Wöchnerinnen und ihre Neugeborenen besonderen kultischer Regelungen unterliegt (Lev I2).

In der Literatur finden sich in Bezug auf diese Regelungen verschiedene Erklärungsansätze, die meist mit den Schlüsselbegriffen der jeweiligen Ritualtheorien in Zusammenhang stehen. Für den Levitikuskommentator Jacob Milgrom ist die Gegenüberstellung von Leben und Tod die zentrale Verstehenskategorie, er sieht hier eine Vermischung von Leben und Tod, während die Aufrichtung der Grenze zwischen Leben und Tod ein zentrales Anliegen des Kults ist. ${ }^{45}$ Howard Eilberg-Schwartz rekurriert auf das Inzesttabu; Mütter und ihre Kinder dürfen nicht zusammen getötet werden. ${ }^{46}$ Humanistische Erklärungsversuche argu-

43 | Vgl. den Begriff "feminized protein«. N. Ruane: Sacrifice, S. 79-84, im Anschluss an Carol Adams.

$44 \mid$ N. Ruane: Sacrifice, S. 82.

45 | J. Milgrom: Leviticus, pass. Hierin folgt inm Th. Hieke: Levitikus, pass.

46 | H. Eilberg-Schwartz: Savage, S. 128-134. 
mentieren mit dem Tierschutz. ${ }^{47}$ Schließlich ist der Ansatz von Nicole Ruane zu nennen, die im Ausschluss der Mutter-Kind-Dyade aus dem rituellen Raum eine Etablierung patrilinearer Bezüge und eine gleichzeitige Unterbrechung der matrilinearen Verbindung sieht.

"Thus, the human impurities of childbirth and ejaculation distance human reproduction from the sacred, whereas the proscriptions aganínst the slaughter of animal parents distance their biological reproduction from it. Sacrificial bonds supersede physical bonds in both the human and animal worlds. " 48

M.E. ist es zur Erklärung dieses Phänomens, des Ausschlusses der Mutter-KindDyade vom rituellen Raum, besonders hilfreich, die von Klawans (s.o.) stark gemachte Verbindung von Reinheit und Opferung heranzuziehen. Dann wird deutlich, dass es nicht um den Ausschluss etwa des spezifisch Weiblichen geht, sondern um Phasen in der Biographie von menschlichen wie tierlichen Müttern und Kindern, in denen diese nur schwer als klar voneinander zu unterscheidende Individuen in den Blick kommen. Dass aber Gegenstände, Pflanzen und tierliche wie menschliche Lebewesen als getrennte Entitäten in den Blick genommen werden können, gehört zu den Grundprinzipien priesterlicher Reinheitsvorstellungen. Nicht umsonst ist der Begriff »trennen« (לדב hi.) ein Leitwort der ersten (priesterschriftlichen) Schöpfungserzählung in Gen I. Taxonomien in Natur und Kultur sind für dieses Denken ausschlaggebend und Mischungen suspekt. Nur wenn die behandelten Gegenstände und Lebewesen voneinander abgrenzbar wahrzunehmen sind, kann eine Ordnung, in der alles an seinem Platz ist, entwickelt werden. Die in Levitikus entfalteten Opferrituale sind eine performative Aktualisierung der Schöpfungsordnung.

"Leviticus has taken sacrifice [...] and made it the framework for a philosophy of life. Sacrifice is one of the main figural motifs with which it presents the principles of God's creation , and the divine order of existence. ${ }^{49}$

Individuen müssen hinsichtlich ihres Geschlechts und anderer physischer Merkmale erkennbar sein. In den ersten Phasen nach der Geburt ist diese Unterscheidung noch nicht möglich, die Mutter-Kind-Dyade stört das Bedürfnis nach klar abgrenzbaren Individuen. Wöchnerin und Säugling bilden eine Einheit, die pränatale Symbiose ist nicht abrupt mit der Geburt zu Ende. Das Wochenbett ist eine Übergangszeit, in der die Trennung von Mutter und Kind geschieht. Diese Übergangszeit wird bei menschlichen Mutter-Kind-Dyaden je nach Geschlecht

47 | Dieses Interpretationsmuster findet sich bereits in der Antike bei Philo von Alexandrien. M. Haran: Kid.

48 | N. Ruane: Sacrifice, S. 103.

49 | M. Douglas: Leviticus, S. 66. 
des Kindes in ihrer Dauer festgelegt. Die Phase der Kultunfähigkeit dauert bei der Mutter eines weiblichen Säuglings doppelt so lange wie nach der Geburt eines männlichen Säuglings (Lev I2) $)^{50}$. Versteht man die Mutter-Kind-Regelungen bei Menschen und Tieren im Horizont der Wichtigkeit von Unterscheidbarkeit, dann wird deutlich, dass die Trennung von der Mutter geschlechtsspezifisch unterschiedlich inszeniert wird. Während das Geschlecht des Mädchens durch eine im Verhältnis zum Jungen doppelt so lange Zeitspanne der Kultunfähigkeit der Mutter markiert wird, fordert die geschlechtliche Bestimmung des Kindes als Jungen ein Beschneidungsritual, das auf seine Weise die Trennung vom mütterlichen Körper kennzeichnet und mit dem Ende der ersten, menstruationsähnlichen Phase der Kultunfähigkeit der Mutter zusammenfällt.

Das Trennungsmotiv kann auch herangezogen werden, um die Opferung der männlichen Erstgeburt zu erklären: Hier wird die Trennung rituell vollzogen und für alle weiteren männlichen Geborenen vorweggenommen. Auch hier wird die Phase der Symbiose beibehalten. Sieben Tage sind Mutter und Kind eine Einheit, erst dann werden sie rituell getrennt behandelt (Ex 22,29-30 vgl. I3,I2-I5 und 34,I9-20.).

Milch symbolisiert die Verbindung von Mutter und Kind, ist Materie, die weder der einen noch dem anderen zugeordnet werden kann. Samuel z.B. wird erst nach dem Abstillen zum Heiligtum gebracht, wenn er also wirklich von seiner Mutter getrennt ist (I Sam I). Diese Trennung muss vollzogen werden, damit überhaupt klar ist, wie weitere Kriterien (Geschlecht, Fehlerlosigkeit) für Opferungen angelegt werden können. Die Mischexistenzen (Wöchnerin, Stillende) bedrohen das Ordnungssystem und ihre jeweiligen Zustände müssen genau geregelt werden. Für die Einordnung in das rituelle System muss eine klare Unterscheidung zwischen Mutter und Kind getroffen werden können.

\section{ZUSAMMENFASSUNG}

Ebenso wenig wie es möglich ist, die Kategorie des Opfers mithilfe eines einzigen Theorierahmens verständlich zu machen, kann der geschlechtsspezifische Aspekt dieses Rituals über einen einzelnen theoretischen Begriff geklärt werden. Wir haben gesehen, dass ökonomische und symbolische Aspekte zusammenwirken, einander überlagern und in ihrer Korrespondenz zu jenen Systemen führen, die die biblischen regulativen Texte in Bezug auf die Opferrituale entwerfen. Außerdem ist deutlich geworden, dass Opfer und Reinheit gemeinsam unter dem Oberbegriff der Heiligkeit betrachtet werden müssen. So können auch Einsichten aus der Analyse der Reinheitsvorstellungen mit den Fragen, die die Opfer aufwerfen, zusammengebracht und für deren Verständnis fruchtbar gemacht werden.

50 | S. dazu ausführlich D. Erbele-Küster: Körper; D. Erbele-Küster: Geschlecht; I. Müllner: Konstruktionen. 
Das Korrespondenzverhältnis menschlicher und tierlicher Ordnungen wird anhand der Opferregelungen und ihrer geschlechtlichen Codierungen in besonders hohem Maß deutlich. Sowohl hinsichtlich ihres Zugangs zum Heiligtum als auch hinsichtlich der Zurückhaltung gegenüber der Mutter-Kind-Dyade im kultischen Raum lassen sich Analogien zwischen den Symbolsystemen, die Tiere betreffen, und den Ordnungen der menschlichen Gesellschaft ausmachen.

Die ritualisierte Tötung von Tieren im Opfer ist also eingebunden in Strukturen der menschlichen Gesellschaft. Sie stützt diese einerseits durch die Regulierung der Gewalt gegenüber Tieren ${ }^{5}$ und andererseits durch die Symbolkraft, die die Opferung von Tieren hat. Gerade in der je nach Opferart unterschiedlichen Spezifizierung der Opfertiere hinsichtlich von Art, Geschlecht und körperlichem Zustand manifestiert sich eine Spiegelfunktion der Tiere in Bezug auf das menschliche Sozialsystem.

\section{LITERATURVERZEICHNIS}

Wenn nicht anders angegeben, dann richten sich die Zitate aus dem biblischen Text nach der Neuen Luther Bibel (I984).

Bark, Franziska: Ein Heiligtum im Kopf der Leser. Literaturanalytische Betrachtungen zu Ex 25-40 (Stuttgarter Bibelstudien, 218), Stuttgart: Verl. Kath. Bibelwerk 2009 .

Becking, Bob: Art.: Jahwe, in: WiBiLex. Online verfügbar unter http://www.bibelwissenschaft.de/stichwort/22127/, zuletzt geprüft am 30.04.2016.

Braulik, Georg: Haben in Israel auch Frauen geopfert? Beobachtungen am Deuteronomium, in: Sigfried Kreuzer/Kurt Lüthi (Hg.): Zur Aktualität des Alten Testaments (FS Georg Sauer), Frankfurt a.M.: Peter Lang I992, S. I9-28.

Braun, Christina von/Stephan, Inge: Gender@Wissen, in: Christina von Braun/ Inge Stephan (Hg.): Gender@Wissen. Ein Handbuch der Gender-Theorien (UTB Gender-Studies, Kulturwissenschaften, Literaturwissenschaften, 2584), Köln: Böhlau 2005, S. 7-45.

Burkert, Walter: Homo necans. The Anthropology of Ancient Greek Sacrificial Ritual and Myth, Berkeley, Calif.: Univ. of California Press i983.

Crüsemann, Frank: Bewahrung der Freiheit. Das Thema des Dekalogs in sozialgeschichtlicher Perspektive, (Kaiser-Taschenbücher, I28), Gütersloh: Kaiser ${ }^{2}$ I998.

51 Inwieweit auch zwischenmenschliche Gewalt durch das Tieropfer reguliert wird, ist umstritten. Vor allem der Ansatz von René Girard und seine besonders in den 80er Jahren des 20. Jahrhunderts intensiv erfolgte und auch vielfach kritisierte Rezeption in der chistlichen Theologie stellt hier einen Zusammenhang her. S. Girard: Das Heilige; N. Lohfink: Gewalt; M. Moosbrugger: Rehabilitierung; R. Schwager: Sündenbock. 
Dahm, Ulrike: Art.: Opfer, in: WiBiLex. Online verfügbar unter http://www.bibelwissenschaft.de/stichwort/24240/, zuletzt geprüft am 30.04.2016.

Dohmen, Christoph: Exodus I-I8 (HThKAT), Freiburg u.a.: Herder 20I5.

Douglas, Mary: Leviticus as Literature, Oxford [u.a.]: Oxford Univ. Press I999.

Douglas, Mary: Purity and Danger. An Analysis of Concept of Pollution and Taboo, London: Routledge (Anthropology) 2002.

Eilberg-Schwartz, Howard: The Savage in Judaism. An Anthropology of Israelite Religion and Ancient Judaism, Bloomington: Indiana Univ. Pr. I990.

Erbele-Küster, Dorothea: Körper und Geschlecht. Studien zur Anthropologie von Leviticus I2 und I5, (Wissenschaftliche Monographien zum Alten und Neuen Testament, I2I), Neukirchen-Vluyn: Neukirchener Verlag 2008.

Erbele-Küster, Dorothea: Geschlecht und Kult. »Rein« und »Unrein« als genderrelevante Kategorien, in: Irmtraud Fischer/Mercedes Navarro Puerto/Andrea Taschl-Erber (Hg.): Tora. (Die Bibel und die Frauen. Eine exegetisch-kulturgeschichtliche Enzyklopädie. Hebräische Bibel - Altes Testament, I), Stuttgart: Kohlhammer 20I0, S. 347-374.

Finsterbusch, Karin: Frauen zwischen Fremdbestimmung und Eigenständigkeit. Genderrelevantes in den Gesetzestexten der Tora, in: Irmtraud Fischer/ Mercedes Navarro Puerto/Andrea Taschl-Erber (Hg.): Tora. (Die Bibel und die Frauen. Eine exegetisch-kulturgeschichtliche Enzyklopädie. Hebräische Bibel - Altes Testament, I), Stuttgart: Kohlhammer 20I0, S. 375-400.

Girard, René: Das Heilige und die Gewalt. Zürich: Benziger I987.

George, Mark K.: Masculinity and its Regimentation in Deuteronomy, in: Creanga, Ovidiu (Hg.): Men and Masculinity in the Hebrew Bible and Beyond, Sheffield: Sheffield Phoenix Press 2010, S. 64-82.

Haran, Menahem: Seething a Kid in Its Mother's Milk, in: JJS 30 (I979), S. 23-35. Hieke, Thomas: Levitikus I-I5 (HThKAT), Freiburg u.a.: Herder 2014.

Klawans, Jonathan: Purity, Sacrifice, and the Temple. Symbolism and Supersessionism in the Study of Ancient Judaism, Oxford/New York: Oxford University Press 2006.

Liss, Hanna: Kanon und Fiktion. Zur literarischen Funktion biblischer Rechtstexte, in: BN I2I (2004), S. 7-38.

Liss, Hanna: Of Mice and Men and Blood. The Laws of Ritual Purity in the Hebrew Bible, in: Hanna Liss/Manfred Oeming (Hg.): Literary construction of identity in the ancient world. Proceedings of the conference Literary fiction and the construction of identity in ancient literatures: options and limits of modern literary approaches in the exegesis of ancient texts, Heidelberg, July I0-I3, 2006. Winona Lake, Ind: Eisenbrauns 20I0, S. I99-2I3.

Lohfink, Norbert: »Gewalt« als Thema der alttestamentlichen Forschung, in: Ders./Ernst Haag (Hg.): Gewalt und Gewaltlosigkeit im Alten Testament (FS Vinzenz Hamp), (Quaestiones Disputatae, 96), Freiburg u.a.: Herder I983, S. I5-50. 
Moosbrugger, Mathias: Die Rehabilitierung des Opfers. Zum Dialog zwischen René Girard und Raymund Schwager um die Angemessenheit der Rede vom Opfer im christlichen Kontext, (Innsbrucker theologische Studien, Bd. 88), Univ., Diss. Innsbruck, Innsbruck, Wien: Tyrolia-Verl. 20I4.

Müllner, Ilse: Pessach als Ereignis und Ritual. Die narrative Einbindung kommender Generationen in Ex I2,I-I3,I6, in: Ute E. Eisen/Peter von Möllendorff (Hg.): Über die Grenze. Metalepse in Text- und Bildmedien des Altertums (Narratologia, 39), Berlin: de Gruyter 2013, S. 59-94.

Müllner, Ilse: Konstruktionen von Geschlecht in regulativen Texten der Tora, in: Christian Frevel (Hg.): Mehr als Zehn Worte. Zur Bedeutung des Alten Testaments in ethischen Fragen (Quaestiones Disputatae 273), Freiburg u.a.: Herder 20I5, S. II5-I52.

Müllner, Ilse: Die Samuelbücher. Gott in Menschen, Tieren und Dingen erzählen, in: Ute E. Eisen/Ilse Müllner (Hg.): Gott als Figur. Narratologische Analysen biblischer Texte und ihrer Adaptionen (HBS 82), Freiburg u.a.: Herder 2016, S. 88-i23.

Petropoulou, Maria-Zoe: Animal Sacrifice in Ancient Greek Religion, Judaism, and Christianity, Ioo BC - AD 200 (Oxford Classical Monographs), Oxford: Oxford Univ. Press 2008.

Ruane, Nicole J.: Sacrifice and Gender in Biblical Law. New York, NY: Cambridge University Press 2013.

Schwager, Raymund: Brauchen wir einen Sündenbock? Gewalt und Erlösung in den biblischen Schriften, München: Kulturverlag I978.

Smith, Jonathan Z.: The Domestication of Sacrifice, in: Ders. (Hg.): Relating Religion. Essays in the Study of Religion, Chicago, Ill.: Univ. of Chicago Press 2004 , S. I45-159.

Steins, Georg: »Ein Gedenken für seine Wundertaten hat er gewirkt«. Exodus I2,I-I5,2I als kulturelles Skript, in: Ilse Müllner/Ludger Schwienhorst-Schönberger/Ruth Scoralick (Hg.): Gottes Name(n). Zum Gedenken an Erich Zenger (HBS 7I), Freiburg u.a.: Herder 2012, S. 85-IO3.

van Wolde, Ellen J.: Reframing Biblical Studies. When Language and Text meet Culture, Cognition, and Context, Winona Lake, IN: Eisenbrauns 2009. 